\title{
DSM 5 - What's new in it??
}

\author{
Subedi S
}

Assistant Professor, Department of Psychiatry, UCMS, Bhairahawa, Nepal

Email Corresponding Author: sandipsubedi@hotmail.com

The fifth edition of Diagnostic and Statistical Manual of Mental Disorders (DSM-5) was published this year, and there was much rejoicing. The changes in this DSM will affect researchers, clinicians, the pharmaceutical industries, the legal systems, and the general public.

The first edition of DSM was published in 1952, the second in 1968, but it wasn't until the DSM-III in 1980 that the psychiatric community could make use of the tool. After the DSM-III, no major revisions were made to the manual, until now. When the DSM-5 planning begun in 1999, there was much written and speculated critics about the magnitude of the changes through use of words like "revolutionary change." A sweeping change based on using a manualwide dimensional model of diagnosis versus the historic traditional categorical model was frequently mentioned. It appears that the categorical model has primarily survived.

The first and most obvious change in the newest version is that the Roman numeral $\mathrm{V}$ has been replaced with the Arabic numeral 5. This change reflects APA's intention to make future revision processes more responsive to breakthroughs in research with incremental updates until a new edition is required. Since the research base of mental disorders is evolving at different rates for different disorders, diagnostic guidelines will not be tied to a static publication date but rather to scientific advances. These incremental updates will be identified with decimals, i.e. DSM-5.1, DSM-5.2, etc., until a new edition is required.

The chapters in DSM 5 is organized reflecting the lifespan approach such that disorders that occur in childhood are in the beginning of the manual and disorders that are seen in older adulthood are mentioned at the end of the manual.

It has moved to a non-axial documentation of diagnosis (Formerly Axes I, II and III), with separate notations for important psychological and contextual factors (Formerly Axis IV) and disability (Formerly Axis V). Clinicians should continue to list medical conditions that are important to understand and manage mental disorders.

It has attempted to integrate scientific findings from the latest research in genetics and neuroimaging. Genetic and physiological risk factors, prognostic indicators, and some putative diagnostic markers are highlighted in the text with the aim to improve clinicians' ability to identify diagnoses in a disorder spectrum based on common neurocircuitry, genetic vulnerability and environmental exposures.

Autistic disorder, Asperger's disorder, and pervasive developmental disorder are consolidated into a single entity "Autism 
spectrum disorder" since the symptoms of these disorders represent the continuum of similar impairments in different severity rather than being distinct disorders.

Unlike previous editions, in DSM 5, classification of bipolar disorder and depressive disorders has been streamlined by including all of the component criteria for each particular disorder (mania, hypomania, major depressive episode, bipolar I disorder, bipolar II disorder) within the respective criteria for respective disorder.

The categories of substance abuse and dependence have been eliminated and replaced with the term "substance use disorder" - with specific substance used, with the hope of alleviating some of the widespread misunderstanding about these issues.

With the advent of various techniques in neuroscience over last 20 years, biological markers identified by imaging for vascular and traumatic brain disorders and specific molecular genetic findings have greatly advanced clinical diagnoses separating these major and mild neurocognitive disorders into specific types in the DSM 5.

The categorical personality disorders are virtually unchanged from the previous edition. However an alternative 'hybrid' model has been proposed in section III guide future research that separates interpersonal functioning assessments and the expression of pathological personality traits for six specific disorders.

A new section (Section III) has been added to highlight disorder that require further study but are not sufficiently well established to be a part of the official classification of mental disorder for routine clinical use. A dimensional measure of symptom severity has also been incorporated. WHO Disability Assessment Schedule (WHODAS) has replaced the Global Assessment of Functioning (GAF) scale.

Apart from these major changes, some changes in the criteria and nomenclature have been made among the pre-existing disorders. DSM 5 has introduced few new disorders like Disruptive Mood Dysregulation Disorder, Excoriation (skin-picking) Disorder, Hoarding Disorder. Details of the change will be clear once we start using the manual in our practice.

As soon as the DSM 5 was published, it has also generated controversy and criticism. Critics, including the National Institute of Mental Health, argue that the DSM represents an unscientific and subjective system There are ongoing issues concerning the validity and reliability of the diagnostic categories; the reliance on superficial symptoms; the use of artificial dividing lines between categories and from 'normality'; possible cultural bias; medicalization of human distress. The publication of the DSM, with tightly guarded copyrights, now makes APA over \$5 million a year, historically adding up to over $\$ 100$ million. Critics have accused the DSM- 5 of lowering diagnostic thresholds across the board, making it far easier for a person to be diagnosed with a mental disorder.

We hope that DSM 5 will be able to satisfy all the users and lives up to the expectations of all those who were involved in the making, and of all those who had been eagerly waiting for the manual since long. 